International Journal of Bifurcation and Chaos, Vol. 28, No. 4 (2018) 1830009 12 pages)

(C) The Author(s)

DOI: $10.1142 /$ S0218127418300094

\title{
Chen System as a Controlled Weather Model - Physical Principle, Engineering Design and Real Applications
}

\author{
Pitikhate Sooraksa \\ Department of Computer Engineering, Faculty of Engineering, \\ King Mongkut's Institute of Technology, Ladkrabang, \\ Bangkok 10520, Thailand \\ pitikhate.so@kmitl.ac.th \\ Guanrong Chen* \\ Department of Electronic Engineering, \\ City University of Hong Kong, P. R. China \\ eegchen@cityu.edu.hk
}

Received January 18, 2018; Revised March 19, 2018

\begin{abstract}
This paper presents the Chen system as a controlled weather model. Mathematically, the Chen system is dual to the Lorenz system via time reversal. Physically, the Chen system can be viewed as a controlled weather model from the anti-control perspective. This paper illustrates the physical principle of this controlled weather model, and develops an engineering design of the model for real indoor climate (temperature-humidity) regulation, with a perspective on outdoor weather control application.
\end{abstract}

Keywords: Chaos; Chen system; Lorenz system; temperature-humidity control; climate control.

\section{Introduction}

The Chen system Chen \& Ueta, 1999] is described by

$$
\begin{aligned}
& \dot{x}=a(y-x), \\
& \dot{y}=(c-a) x-x z+c y, \\
& \dot{z}=x y-b z,
\end{aligned}
$$

where, to generate chaos, typical parameters are $c=28, a=35$, and $b=3$. It was originally derived from anti-control of the classical Lorenz system Lorenz, 1963]

$$
\begin{gathered}
\dot{x}=a(y-x), \quad \dot{y}=c x-x z-y, \\
\dot{z}=x y-b z,
\end{gathered}
$$

where typical parameters are $c=28, a=10$, and $b=8 / 3$. The so-called "anti-control of chaos" was meant to introduce a control input to make an originally nonchaotic system become chaotic Chen, 1997]. Here, a simple linear anti-controller of the form $u=\alpha x+\beta y+\gamma z$ was applied to the righthand side of the second equation of the Lorenz equation (2), which had the three parameters originally not in the chaotic region, to try to generate (new) chaotic dynamical behaviors. Based on the wellknown criterion of Shilnikov [1965], it was found that the anti-controller could be chosen as

$$
u=-a x+(1+c) y+0 z
$$

thereby yielding the Chen system (10).

\footnotetext{
*Author for correspondence

This is an Open Access article published by World Scientific Publishing Company. It is distributed under the terms of the Creative Commons Attribution 4.0 (CC-BY) License. Further distribution of this work is permitted, provided the original work is properly cited.
} 


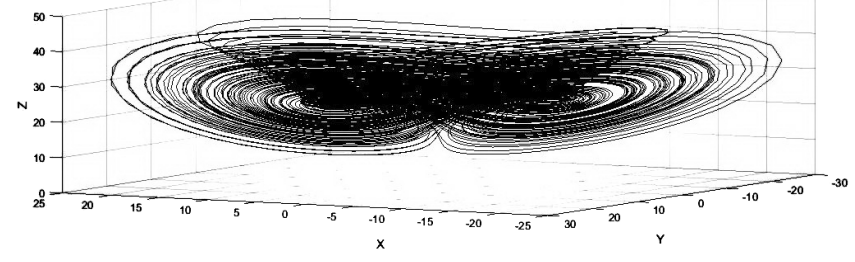

(a)

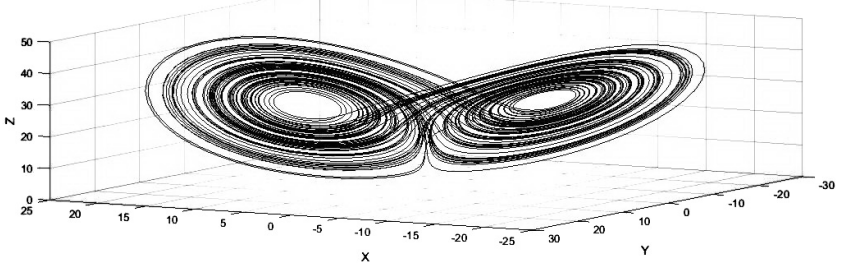

(b)

Fig. 1. Comparison of two chaotic attractors. (a) Chaotic Chen attractor and (b) chaotic Lorenz attractor.

As a side note, by similarly choosing $u=$ $-c x+(1+c) y+0 z$, one obtains the Lu system Lu \& Chen, 2002

$$
\begin{gathered}
\dot{x}=a(y-x), \quad \dot{y}=-x z+c y, \\
\dot{z}=x y-b z,
\end{gathered}
$$

where typical parameters are $c=22.2, a=30$, and $b=44 / 15$.

By comparing the chaotic attractors of the Chen system to the Lorenz system, generated with the same initial condition $x_{0}=-3, y_{0}=2$, $z_{0}=20$ as shown by Fig. 1, the new attractor moves upward much more fiercely, prominently driven by the internal force governed by the new input (3) to the system equations. This paper illustrates the physical principle and mechanism that create this difference in their chaotic dynamics, and explains why some seemingly simple modifications of the Lorenz system provide drastically different dynamics. It will be further shown that the new dynamical mechanism embedded in the Chen system, which by nature is a controlled Lorenz system, could be useful as an engineering design for some real applications, with two examples presented on indoor climate (temperature-humidity) regulation and a perspective on outdoor weather control application.

The rest of the paper is organized as follows. Section 2 illustrates the physical principle of the Chen system developed from the Lorenz system. Section 3 develops an engineering design utilizing the new characteristics of the Chen system. Section 4 shows two examples of indoor and outdoor climate controls. Section 5 concludes the paper with an outlook on potential future applications.

\section{Physical Interpretation}

Similarly to the Lorenz system, which is a simplified weather dynamics model, the parameters in the Chen system (11) can be interpreted as follows Rohsenow et al., 1985]:

- $x$ is a variable of the spatial average of hydrodynamic velocity, which is proportional to the intensity of the fluid convective motion;

- $y$ is a variable of temperature, which is the temperature difference between the ascending and descending currents;

- $z$ is a variable of the temperature gradient or deviation, which is proportional to the distortion of the vertical temperature profile from linearity;

- dimensionless positive constant $a$ is the Prandtl number;

- dimensionless positive constant $c$ is the Rayleigh number, which is the product of the Grashof number and the Prandtl number, therefore $a$ and $c$ are mutually dependent;

- dimensionless positive constant $b$ is related to the ratio of the spatial dimensions involved.

Recall that the Prandtl number $(\mathrm{Pr})$ is the ratio of momentum diffusivity to thermal diffusivity. A smaller Pr value implies a more rapid heat diffusion compared to the momentum, whereas a larger Pr value means that the momentum becomes more diffusivity dominant on the dynamics. Recall also that the Grashof number is defined as the ratio of the buoyancy force to the viscous force acting on the fluid, which often arises in the study of free convection or natural convection. And, the Rayleigh number can be described by the product of these two ratios. In the atmospheric convection model, the Rayleigh number is proportional to the difference in the temperatures from the warm base to the cool top in a convective cell. Unlike the Prandtl number, both the Grashof number and the Rayleigh number depend on the differences between the surface temperature and the bulk temperature, as well as on a characteristic length in the model.

Although the variables and parameters in Eqs. (10)-(3) are dimensionless after reasonable 
mathematical simplifications, their corresponding physical properties are preserved, which are further discussed below. Specifically, in the following, variable $x$ is considered as the air flow, $y$ as the temperature, and $z$ as the relative humidity. The $z$ variable represents a cross-coupling effect and is inherently a fluctuated variable of the temperature. Usually, temperature affects humidity, namely the water vapor or humidity is limited by the temperature in a parcel of air. In fact, humidity responds to temperature in such a way that with higher temperature the air holds more water vapor. Note, however, that humidity has a rather different temperature profile in the vertical direction in space.

Now, consider the Lorenz system (2), with control input (3) being added to the right-hand side of its second equation, leading to the Chen system (11).

Physically, the change of the temperature, namely the term $\dot{y}$ in the Lorenz system (2), is being modified by the control input (3):

- the first term of the control input actually adds a negative driven flow;

- the second term of the control input adds more heat flux into the second equation of the system.

As a result, the free convective motion in the original Lorenz system becomes a forced convection process. As pointed out by Barboza [2018], the second equation of the Chen system (11) is regenerative, characterized by the positive-feedback term $+c y$ in the controller (3), which is a fundamental property of the Chen system distinguishing itself from the Lorenz system.

More precisely, keeping in mind the magnitudes of the parameters and signs of the variables in the control input (3), the first term - ax can be interpreted as reversing the direction of the air flow, which reduces the rate of temperature change along the $x$-axis scaled by a factor of $a$. The second term $(1+c) y$ can be interpreted as modifying the temperature by adding more heat flux to the system, scaled by a factor of $(1+c)$. The schematic diagram shown in Fig. 2 illustrates the underlying physical process.

It is remarked that. as pointed out bv Leonov and Kuznetsov 2015], for physical realization of the control usually it is necessary to have bounded control, i.e. dissipativity in the sense of Levinson (having an absorbing set); while it is trivial for the Lorenz system, which has an absorbing set for any parameter used, it is nontrivial for the Chen system (1) and the Lu system (4).

Numerically, the magnitudes of the system parameters $(a, b, c)$ of the Chen system are $(35,3$, $28)$ and that of the Lorenz system are $(10,8 / 3,28)$. Thus, the Prandtl number of the Chen system is higher than that of the Lorenz system. More importantly, in the Chen system the direction of the increasing temperature, $+c y$, is in the opposite direction with a greater magnitude of the Rayleigh number $(c \gg 1)$ compared to that in the Lorenz

COLD AREA

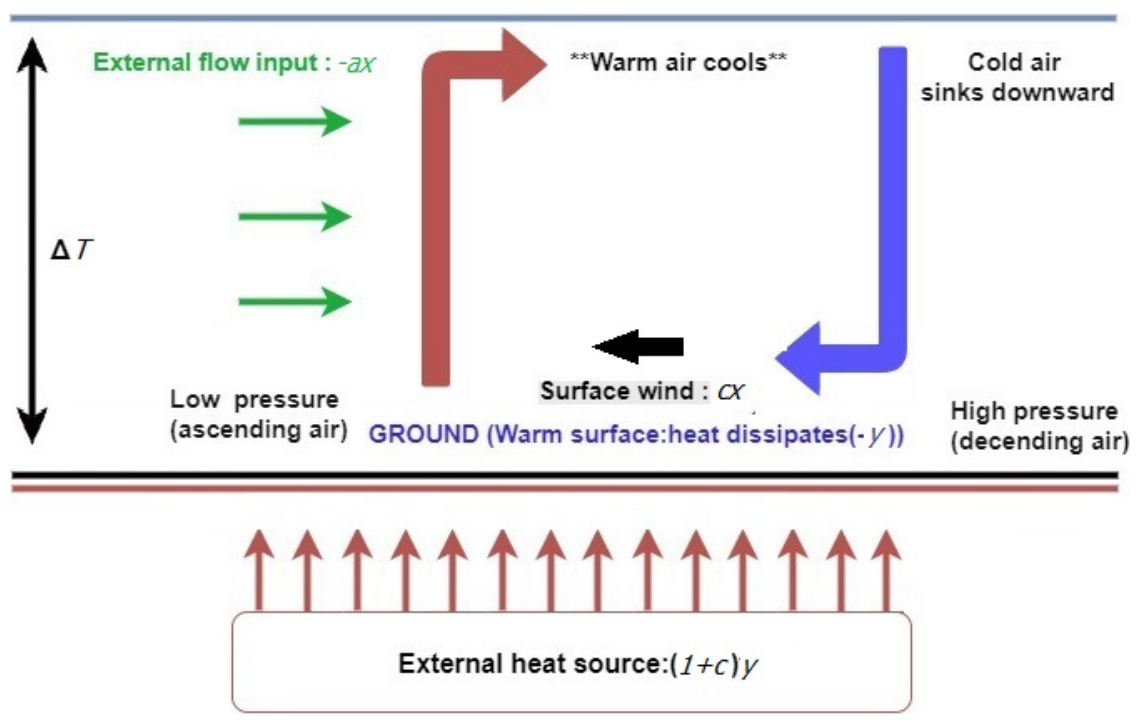

Fig. 2. Physical process of the controlled air convection. 
system. $-u$. which was alreadv pointed out bv Barboza [2018], as mentioned above.

By nature, the Chen system describes a state of severe conditions of the weather system, where the temperature and its fluctuation and therefore the air flow are all higher than those of the Lorenz system, verified also by its larger maximum Lyapunov exponent Ueta \& Chen, 2000].

Now, imagine that a storm is forming from the Lorenz system, and an external control force (e.g. heated air) is being injected into the air from the ground. Effectively, the external air-flow and heatflux input will force the original air flow to move upward much more fiercely, resulting in a hotter and more humid air convection system as a whole, with a much stronger momentum of flow motion. Besides, the air flow is contaminated with various types of particles besides water vapors $(a=\operatorname{Pr}$ of water ranges only within the scale of 1-10). All these together generates the drastically rising hat in the center of the chaotic Chen attractor from the originally flat butterfly-shaped Lorenz attractor (see Fig. 1 again for a visual comparison).
Note that the above control effects can be realized physically. Indeed, it has been known [Landahl \& Mollo-Christensen, 1992] that applying gradient pressure to disturb a flow can be done by ejecting a rapid outflow of a low-speed fluid from the surface region into the inflow of a high-speed fluid. This can create a shear flow that eventually yields turbulence, as illustrated by Fig. 3(a). And, applying a heat flux of a high Rayleigh number to a flow by heating the horizontal surface can likewise create a turbulent flow of the fluid, as illustrated by Fig. 3(b). Now, it can be easily imagined that, under the combined effect of the above two actions, the regular cellular air-flow structure in the air will disappear and a fully turbulent flow will take place. As a matter of fact, this phenomenon has been repeatedly observed, for example in The Burning Man art faslv007, 2003; Jukin Media, 2016] and even in tornado formations.

As shown in the Appendix, the above controlled Lorenz system, namely the Chen system, is both linearly controllable and observable, and it possesses a minimum realization, namely, $x, y$ and $z$



(a)


(b)

Fig. 3. Turbulence forming processes: (a) Conceptual illustration of progression of flow formation of span-wise vortices (Fig. 8.5 on p. 111 in Landahl \& Mollo-Christensen, 1992]) and (b) thermals rising from a heated horizontal surface (Fig. 11.2 on p. 146 in [Landahl \& Mollo-Christensen, 1992]). 
are minimal to describe the controlled weather system. These nice properties of the Chen system make its engineering design possible, towards a controlled weather system for some real and potential applications. This will be further discussed next.

\section{Engineering Design for Indoor Climate Control}

Consider a familiar HVAC (Heating, Ventilation, and Air Conditioning) system. The objective here is to design, based on the typical HVAC system, an indoor climate control system comprising a humidifier unit controlled by an ultrasonic circuit to automatically adjust the relative humidity $(\mathrm{RH})$ to be beneficial for human health and comfort.

It has been known for quite a long time ANSI/ASHRAE, 2012] that, in spaces with air speed less than $0.2 \mathrm{~m} / \mathrm{s}$, the $\mathrm{RH}$ within the range of $40<\mathrm{RH}<60$ and the temperature within the range of $22-27^{\circ} \mathrm{C}$ together provide a comfort zone for humans and, meanwhile, this condition can eliminate pathogens (e.g. viruses, bacteria, funguses) and allergic substances as well as reducing or even eradicating house dust mites Arlian et al., 2001; Moungthong et al., 2014; Sookchaiya et al., 2010.

To design a good sanitizing room, such as a surgery room that requires precise climate control, a conceptual control system model is needed. First, observe from Eq. (1) or (2) that the flow, the temperature and the $\mathrm{RH}$ are described by the nonlinear cross-coupling terms. A typical control design strategy is to decouple these variables for easier analysis and computation. To do so, one could first set the fan speed to constant; for example, fix the wind speed to be at the desired value of $0.2 \mathrm{~m} / \mathrm{s}$, which will continuously provide uniform ventilation to a concerned area in the room (or the entire room if it is relatively small). As a result, the constant wind speed gradually leads the temperature and $\mathrm{RH}$ to constant in the concerned area, so that the two wind-speed-dependent dimensionless variables $x \approx y$ in the vector field of the Chen system (1), yield

$$
\dot{x}=0, \quad \dot{y}=-a y-y z, \quad \dot{z}=y^{2}-b z .
$$

Although both temperature and $\mathrm{RH}$ in the dimension-reduced equation (5) are only approximated, this reduced-order system provides a good model for designing an effective control strategy for the original system. In fact, this kind of complexityreduced controller design methods are familiar to engineers as a practical approach.

Now, the third equation in (5) suggests that changing the temperature is more sensitive than changing the $\mathrm{RH}$ of the system, as the input variable on the right-hand side of the equation is in a parabolic form. This verifies the psychometric chart shown in Fig. 4, the ANSI/ASHAE Standard 55-2010, to which Eq. (5) is applicable. As the temperature increases by around $3^{\circ} \mathrm{C}$, the $\mathrm{RH}$ is correspondingly changed by roughly $9 \%$, for the region within the comfort zone.

It is remarked that although more accurate but also more complex models are available for analysis Bosen, 1958; Romps, 2014], which depend even on the height above the Earth's surface, geographic region, air pressures and other related factors, for the objective of climate control to track a desired temperature, $T_{d}$, and a desired $\mathrm{RH}, \mathrm{RH}_{d}$, Eq. (5) is adequate and good enough, as will be seen from the real application of an indoor climate control system to be further discussed below. A parallel control architecture for the above decoupling model is presented in Fig. 5

It is also remarked that, through the indoor climate control process, once the desired temperature $T_{d}$ is attained, which can be automatically accomplished by using an inverter control unit, one can turn on the heater and the humidifier so as to control the $\mathrm{RH}$ to reach the set point $\mathrm{RH}_{d}$. This can be accomplished by setting an interval of tolerance, $\Delta$, around $T_{d}$. If, during the process, the value $T$ falls into the interval of $T_{d} \pm \Delta$, the RH controller is activated within its operational range. If $T$ is not within the designed tolerance region, the inverter control unit is activated to enforce it to move to the set point. Once the temperature is settling into the set-point region, the cycle of $\mathrm{RH}$ control is activated again. Mathematically, from Eq. (5)), the desired temperature $T_{d}$ is reached when $\dot{y}=0$. Consequently, it is easy to solve the equation for $z$, for $T \in\left[T_{d}-\Delta, T_{d}+\Delta\right]$.

Next, to design and implement a real indoor climate control system, two benchmark models are reviewed to reveal some basic ideas. In [Moungthong et al., 2014], a $42 \mathrm{~m}^{3}$ room is used to install an indoor climate control system. The set-point values are $T_{d}=25^{\circ} \mathrm{C}, \Delta=0.5$, and $\mathrm{RH}_{d}=55 \%$. The room is fumed with a large amount of dangerous fungus called Aspergillus flavus (A. flavus) and 




Fig. 4. A comfort zone defined by ANSI/ASHAE Standard 55-2010 ANSI/ASHRAE, 2012].

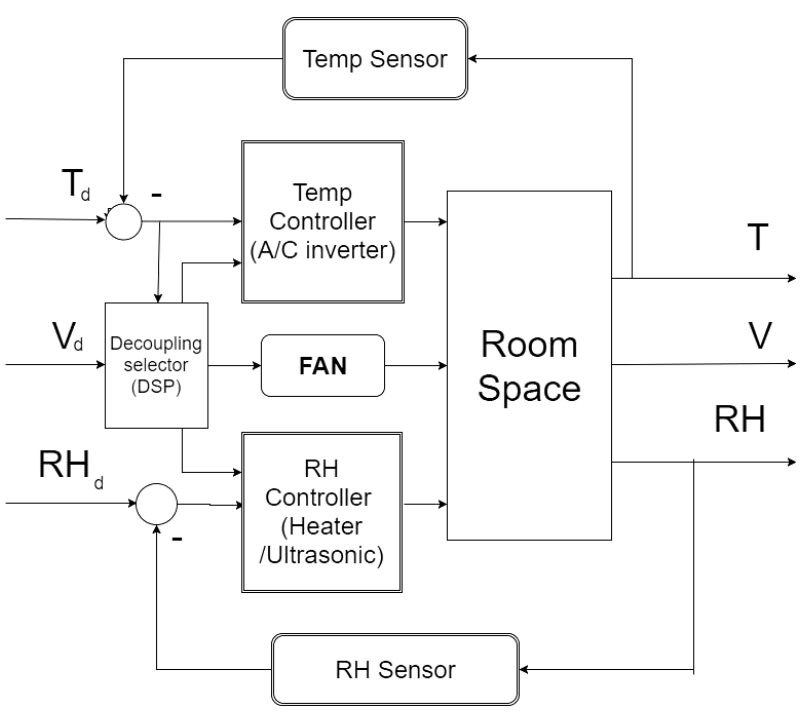

Fig. 5. The conceptual decoupled system model for indoor climate control. the room is closed for 20 days. The climate control results demonstrated that, for the first three days, there are nine $\mathrm{A}$. flavus colonies found in the room. However, after six days, nine days, 12 days and 15 days, the air samples contain neither fungus nor bacteria. In Sookchaiya et al., 2010], two $20 \mathrm{~m}^{2}$ rooms are used for testing. One is a classroom and the other is a bedroom. The desired $\mathrm{RH}$ value for both cases is set to be within the interval $[55 \%, 60 \%]$, with $T_{d}=25^{\circ} \mathrm{C}$ and tolerance $\Delta=$ $\pm 0.2^{\circ} \mathrm{C}$ for the classroom and $\pm 0.35^{\circ} \mathrm{C}$ for the bedroom. Satisfactory results were reported for both cases.

Now, an engineering design and practical implementation of a real indoor climate control system based on the mathematical principles of the controlled Lorenz system (2), namely the Chen system (1), is illustrated. 


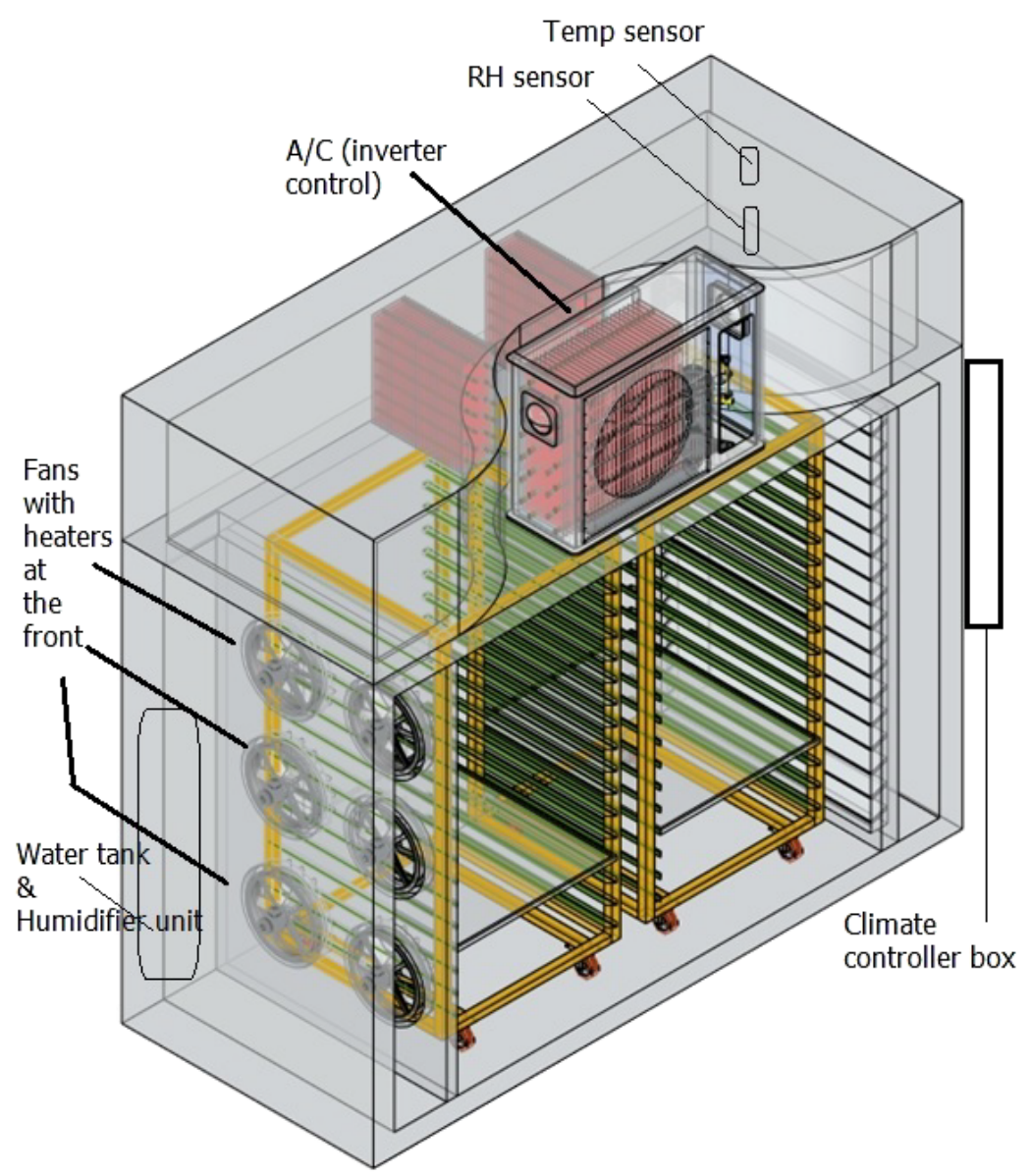

Fig. 6. The implemented climate control system for drying agricultural products.

Consider climate control system used for drying agricultural products, as shown by Fig. 6, with technical data for measuring instrument and component specification as given in Table 1]. This system was built in a laboratory located in the King Mongkut's Institute of Technology, Ladkrabang, Bangkok, Thailand. The control system was implemented based on the structure shown in Fig. 5, which was designed following the Chen system model as discussed in detail above.

Table 1. Control system instrument/components and measurement specification.

\begin{tabular}{lcc}
\hline Instrument/Component & $\begin{array}{c}\text { Measurement/ } \\
\text { Specification }\end{array}$ & Accuracy \\
\hline Anemometer & Air velocity & $\pm 1 \%$ \\
Temperature sensor & RTD (Pt100 X) & $\pm 0.05^{\circ} \mathrm{C}$ \\
RH sensor & STH15 & $\pm 0.1 \%$ \\
A/C (inverter control) & $12000 \mathrm{BTU}$ & - \\
Heater & $2 \mathrm{~kW}$ & - \\
Humidifier (ultrasonic) & $200 \mathrm{ml} / \mathrm{h}$ & - \\
\hline
\end{tabular}

In the chamber, coffee beans from the fermentation process are filled into each tray in a $1 \mathrm{~m}$ width $\times 1.2 \mathrm{~m}$ length $\times 1.8 \mathrm{~m}$ height compartment, to be dried to be of $11-12 \%$ moisture content. The control strategy discussed above is applied, as illustrated by Fig. 7, which presents both temperature and $\mathrm{RH}$ inside the chamber during the process. The desired $\mathrm{RH}, \mathrm{RH}_{d}$, is within $[50 \%, 60 \%], T_{d}=25^{\circ} \mathrm{C}$, and $\Delta= \pm 0.5^{\circ} \mathrm{C}$. The desired wind speed, $V_{d}$, is fixed at $0.5 \mathrm{~m} / \mathrm{s}$.

It can be observed from Fig. 7 that there are peaks of the $\% \mathrm{RH}$ output appearing periodically. This is because the decoupling strategy allows the temperature controller to turn on the control input whenever the temperature exceeds the allowable tolerance limit. The RH control loop is activated again once the temperature is within the desired range.

Experiments have confirmed that qualitatively the dried coffee beans preserve aroma, chlorogenic acid, color and other temperature-sensitive substances. Statistical comparisons have also confirmed 


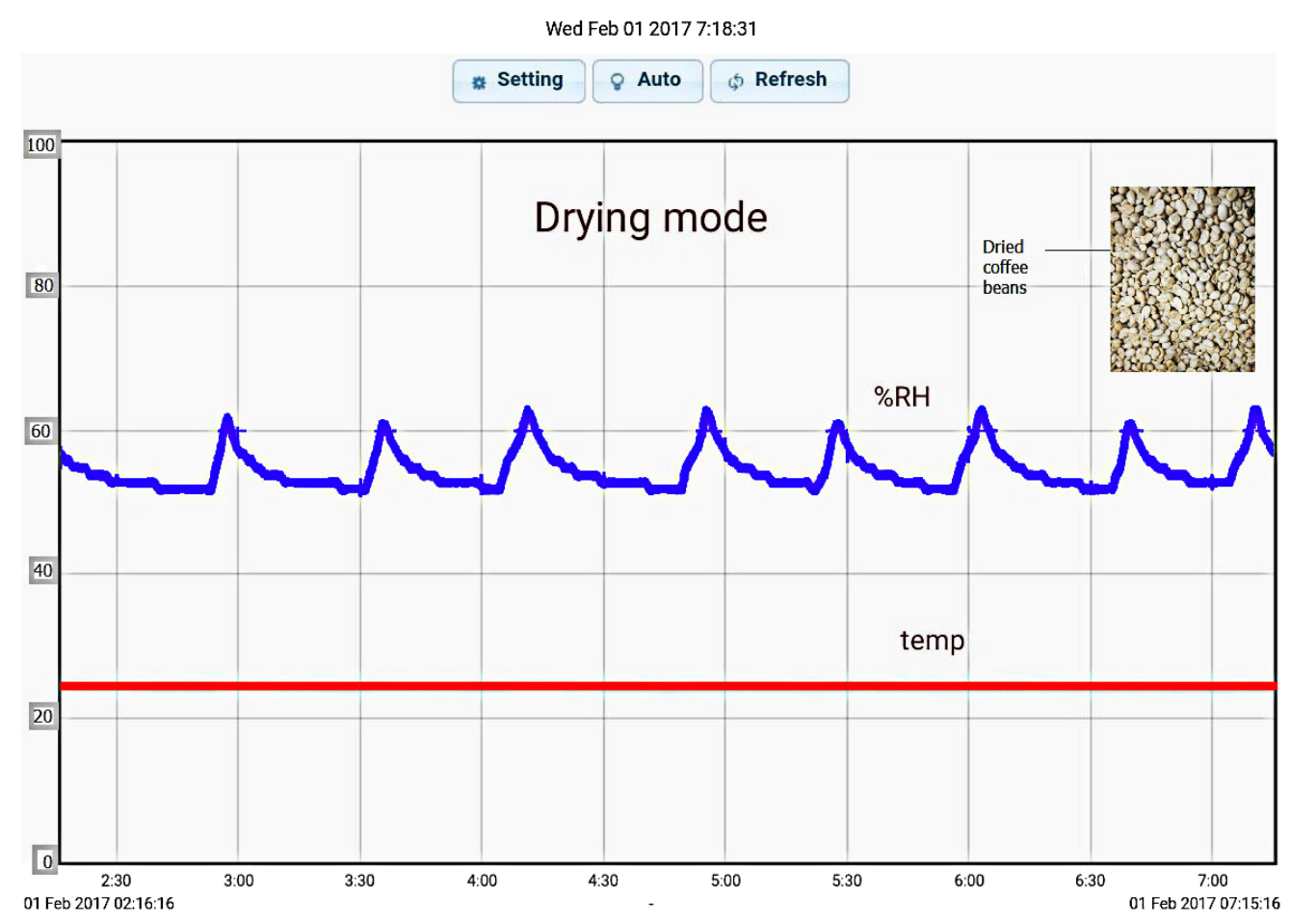

Fig. 7. Temperature and RH of the control system during the process (time unit is hour:minute).

that using the designed climate control system provides better results than that using the conventional means of sun- or wind-drying treatments of agricultural products like coffee beans.

\section{Future Application in Outdoor Weather Control}

This section illustrates how an application of the controlled Lorenz system, namely the Chen system, to outdoor weather control is possible. From a control-theoretic perspective, this section provides an anti-control conceptual framework for outdoor weather modification.

Historically, an experiment was conducted by Schaefer [1946] via spraying dry ice into a freezer containing supercooled droplets, which was cloud droplets being cooled well below $0^{\circ} \mathrm{C}$. As a result, ice crystals were formed, which could be considered as weather modification. Later, it was also experimented by Langmuir [1948] via spraying silver iodide onto cold cloud at temperatures below the water vapor's freezing point, which made the ice crystals grow bigger. The droplets then were solidified faster and the size was large enough to fall out of the cloud. As the temperature increased, the ice melted and became rain. More reports about weather modification techniques can be found in the book by Cotton and Pielke [2007].

A simple, effective, and easy-to-understand demonstration of how the Lorenz system can be transformed to be the Chen system is perhaps the rainmaking technique suggested by the King of Thailand, His Majesty the King Bhumibol Adulyadej 2005], which was invented in 1969 iamtube002, 2009].

Specifically, with great concern for the drought problem due to delayed seasonal rains once in Thailand, an artificial rainmaking technique was proposed by the Thailand King as illustrated by Fig. 8 . For a brief introduction, the procedure of rainmaking is summarized into three operational steps, i.e. agitating, ripening and disturbing, respectively, as follows.

\section{Step 1. Agitating}

This step helps in forming rain clouds. The goal is to create humidity by a mass of air rising. Hygroscopic chemicals such as $\mathrm{NaCl}$ are used for stimulating cloud forming (Condition \#1 in Fig. 8). From a scientific point of view, this step changes the values of $\operatorname{Pr}$ (i.e. the parameter $a$ ) and the Ra number $c$ in the weather system within the targeted area, so as to increase the amount of potential rainfall. 


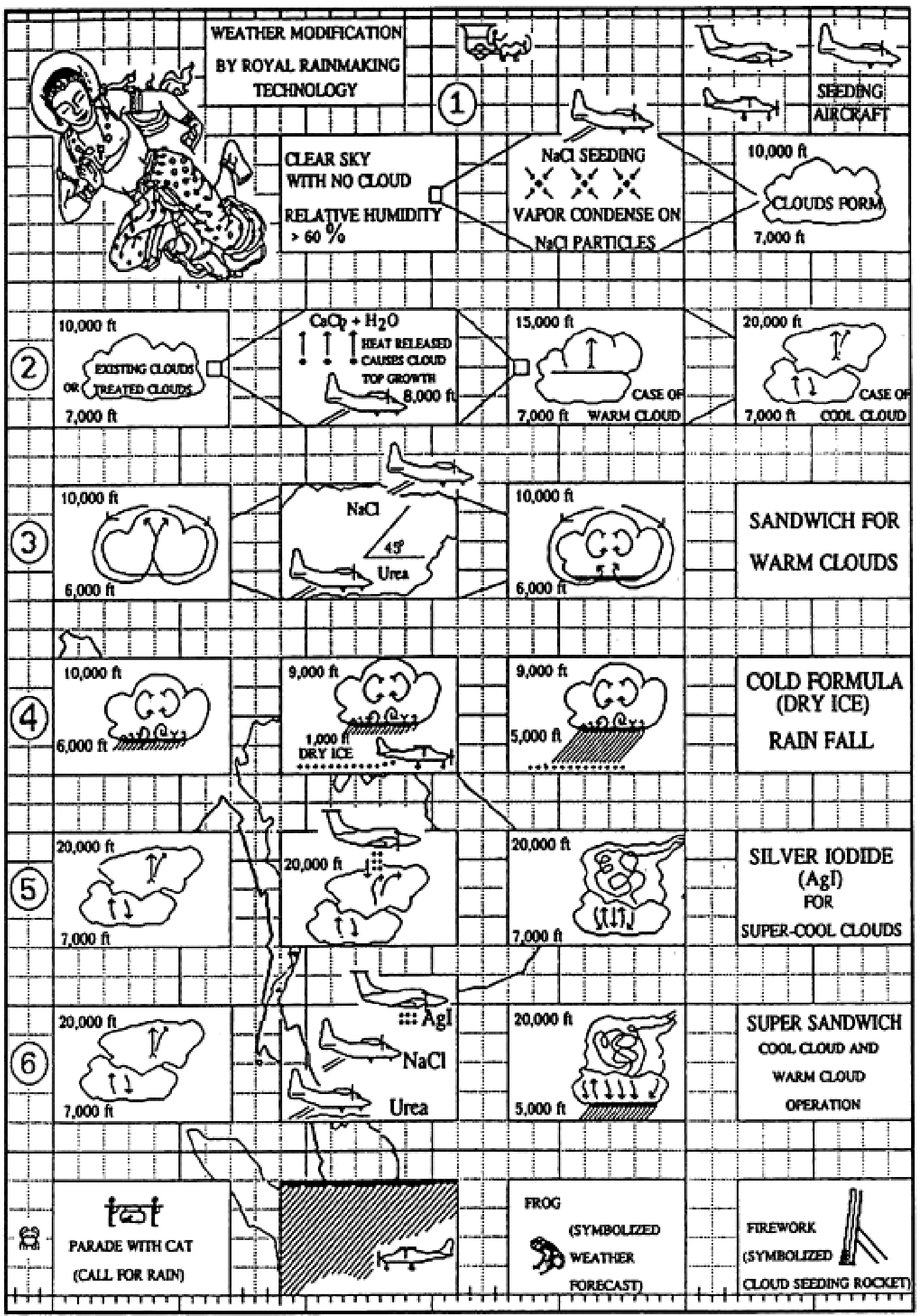

Fig. 8. Procedure of rainmaking for weather modification [His Majesty the King Bhumibol Adulyadej, 2005]. 
Step 2. Ripening

This step makes the droplets of water to be more condensed by scattering exothermic hygroscopic chemicals such as $\mathrm{CaCl}$ and water in Condition \#2, $\mathrm{NaCl}$ and Urea in Condition \#3, dry ice in Condition \#4, silver iodide in Condition \#5, and $\mathrm{AgI}+\mathrm{NaCl}+$ Urea in Condition \#6, respectively. All conditions are formulated to use hygroscopic chemicals for heating and cooling, which cause a significant temperature difference between the upper layer and the lower layer of the cloud, resulting in the change of the Ra number $c$. Mathematically, it is the action that the term $(1+c) y$ in the control input (3) is introduced into the original Lorenz system, thereby converting it to the Chen system. This is in accordance with the description in Langmuir, 1948] that the latent heat released as ice crystals grown by vapor deposition would warm the seeded part of the cloud, causing upward air-flow motion leading to turbulence. In addition, many pieces of cloud are buoyancy-driven. When an air parcel gets heated, this volume of air becomes warmer and so expands. The warmed air will be buoyed up in the upward direction, affecting the change of Ra and $\mathrm{Pr}$ in reality model. The expansion then changes the geometric shape of the cell, yielding a change of the constant $b$ in Eq. (2), reminiscent of the description in Siddheshwar \& Titus, 2013].

\section{Step 3. Disturbing}

This step helps expedite the process of rain-falling by flying one or more airplanes through the heavy clouds, which is equivalent to creating a shear flow, the term $-a x$ in the control input (3), and injecting it to the original Lorenz system.

More details on modifying weather conditions for rainmaking can be found in the three-volume project report Silverman et al., 1994].

\section{Conclusion}

This article interprets the chaotic Chen system as a controlled Lorenz system with significant physical meanings of the control input terms, which can clearly explain some commonly observed natural phenomena and lead to an engineering design with practical implementation of a real indoor climate control system, showing a foreseeable potential in future applications to outdoor weather modification at various scales. Looking forward, the Chen system as a controlled weather system model may even suggest military applications such as in heating electron clouds in the ionosphere to create thunderbolts and powerful pulse waves, which can provide tremendous amounts of heat and wind shears [Bailey \& Worthington, 1997]. Such conditions could potentially lead to man-made hurricanes or tornadoes on the Earth, for better or for worse, which should be pursued or be prohibited by the scientific and engineering communities for good humanitarian reasons.

\section{Acknowledgments}

This work is dedicated to His Majesty the King Bhumibol Adulyadej for his leadership and contributions to weather modification by his royal rainmaking technology as his legacy to Thailand and the world. Many thanks to Dr. V. Monyakul for providing technical data and valuable suggestions on the indoor climate controller design and implementation. This work is financially supported by the National Research Council of Thailand under the Grants 2557 and 2560, and the Chaotic Climate Control Project sponsored by the Faculty of Engineering, King Mongkut's Institute of Technology, Ladkrabang, Thailand, as well as by the Hong Kong Research Grants Council under the GRF Grant CityU11200317.

\section{References}

ANSI/ASHRAE [2012] "ANSI/ASHRAE Standard 55-2010," Thermal Environmental Conditions for Human Occupancy (American Society of Heating Refrigerating and Air-Conditioning Engineers Inc., Atlanta, USA).

Arlian, L. G., Neal, J. S., Morgan, M. S., VyszenskiMoher, D. L., Rapp, C. M. \& Alexander, A. K. [2001] "Reducing relative humidity is a practical way to control dust mites and their allergens in homes in temperate climates," J. Allergy Clin. Immunol. 107, 99-104.

Bailey, P. G. \& Worthington, N. C. [1997] "History and application of HAARP technology: The high frequency active auroral research program," Proc. 32nd Intersociety Energy Conversion Engineering Conf., doi: 10.1109/IECEC.1997.661959.

Barboza, R. [2018] "On Lorenz and Chen systems," Int. J. Bifurcation and Chaos 28, 1850018-1-8.

Bosen, J. F. [1958] "An approximate formula to compute relative humidity from dry bulb and dew point temperature," Monthly Weather Rev. 86, 486.

Chen, G. [1997] "Control and anti-control of chaos," Proc. 1st Conf. Oscillations and Chaos, St. Petersburg, Russia, 27-29 August 1997, pp. 181-186. 
Chen, G. \& Ueta, T. [1999] "Yet another chaotic attractor," Int. J. Bifurcation and Chaos 9, 1465-1466.

Chui, C. K. \& Chen, G. [1989] Linear Systems and Optimal Control (Springer-Verlag).

Cotton, W. R. \& Pielke, R. A. [2007] Human Impacts on Weather and Climate, 2nd edition (Cambridge University Press).

fasly007 [2003] Dust Devils @ Burning Man 2013. Published on September 10, 2013. Retrieved on January 1, 2018 from: https://www.youtube.com/watch?v=MEyP1ebYs0.

His Majesty Bhumibol Adulyadej [2005] "Weather modification by royal rainmaking technology," US Patent, US20050056705 A1.

iamtube002 [2009] King of Thailand Raindrops from Royal Generosity. Retrieved on January 9, 2018 from: https://www.youtube.com/watch?v=Rxd3xfR2jZk.

Jukin Media [2016] Fiery Dust Tornado at Burning Man. Published on September 13, 2016. Retrieved on January 1, 2018 from: https://www.youtube.com/watch? $\mathrm{v}=$ YHR3WJn3I6M.

Landahl, M. T. \& Mollo-Christensen, E. [1992] Turbulence and Random Processes in Fluid Mechanics, 2nd edition (Cambridge University Press).

Langmuir, I. [1948] "The production of rain by a chain reaction in cumulus clouds at temperatures above freezing," J. Atmosph. Sci. 5, 175-192.

Leonov, G. A. \& Kuznetsov, N. V. [2015] "On differences and similarities in the analysis of Lorenz, Chen and Lu systems," Appl. Math. Comput. 256, 334-343.

Lorenz, E. N. [1963] "Deterministic nonperiodic flow," J. Atmosph. Sci. 20, 130-141.

Lu, J. \& Chen, G. [2002] "A new chaotic attractor coined," Int. J. Bifurcation and Chaos 12, 659-661.

Moungthong, G., Klamkam, P., Mahakit, P., Chalermwatanachai, T., Thunyaharn, S. \& Monyakul, V. [2014] "Efficacy of the precise climate controller on the reduction of indoor microorganisms," Asia Pacific Allergy 4, 1-6.

Rohsenow, W. M., Hartnett, J. P. \& Ganic, E. N. [1985] Handbook of Heat Transfer Fundamentals, 2nd edition (McGraw-Hill).

Romps, D. M. [2014] "An analytical model for tropical relative humidity," J. Climate 27, 7432-7449.

Schaefer, V. J. [1946] "The production of ice crystals in a cloud of supercooled water droplets," Science $\mathbf{1 0 4}$, $457-754$.

Shilnikov, L. P. [1965] "A case of the existence of a denumerable set of periodic motions," Soviet Math. Dokl. 6, 163-166.

Siddheshwar, P. G. \& Titus, P. S. [2013] "Nonlinear Rayleigh-Bénard convection with variable heat source," J. Heat Transfer 135, 122502.

Silverman, B. A., Hartzell, C., Woodley, W. L. \& Rosenfeld, D. [1994] Thailand Applied Atmospheric
Research Program, Vols. 1-3 (U.S. Department of the Interior, Bureau of Reclamation).

Sookchaiya, T., Monyakul, V. \& Thepa, S. [2010] "Assessment of the thermal environment effects on human comfort and health for the development of novel air conditioning system in tropical regions," Energy and Buildings 42, 1692-1702.

Ueta, T. \& Chen, G. [2000] "Bifurcation analysis of Chen's equation," Int. J. Bifurcation and Chaos 10, 1917-1931.

\section{Appendix}

\section{Controllability Matrix and Observability Matrix of the Linearized Chen System}

Assume that the concerned weather system is evaluated in an infinitesimal region on an infinitesimal time scale, such that the incremental changes in the system dynamics are nearly linear. This leads to the system matrices and vectors equations (A.1)(A.3), with variables $x, y$, and $z$ representing the flow, temperature, and relative humidity as discussed above in the text. The input $u$ in Eq. A.1 is a feedback control term specified as in (A.3), which applies external flow and temperature to the original Lorenz system, leading to the Chen system as a controlled Lorenz system.

$$
\begin{aligned}
\frac{d}{d t} X & =\left[\begin{array}{ccc}
-a & a & 0 \\
c-z & -1 & 0 \\
y & 0 & -b
\end{array}\right] X+\left[\begin{array}{l}
0 \\
1 \\
0
\end{array}\right] u(x, y, z), \\
Y & =X=\left[\begin{array}{lll}
1 & 0 & 0 \\
0 & 1 & 0 \\
0 & 0 & 1
\end{array}\right]\left[\begin{array}{l}
x \\
y \\
z
\end{array}\right]=\left[\begin{array}{l}
x \\
y \\
z
\end{array}\right], \\
u & =\left[\begin{array}{lll}
-a & 1+c & 0
\end{array}\right] \quad X=-a x+(1+c) y .
\end{aligned}
$$

Recall Chui \& Chen, 1989 that the Controllability Matrix (CM) of the above linearized system is defined by $\mathrm{CM}=\left[\begin{array}{lll}B & A B & A^{2} B\end{array}\right]$, which is

$$
\mathrm{CM}=\left[\begin{array}{ccc}
0 & a & -a(a+1) \\
1 & -1 & a(c-z)+1 \\
0 & 0 & a y
\end{array}\right] .
$$

Since the matrix CM has a full-row rank, the system is controllable. 
Similarly [Chui \& Chen, 1989], the Observability Matrix (OM) of the system is given by

$$
\mathrm{OM}=\left[\begin{array}{c}
C \\
C A \\
C A^{2}
\end{array}\right]=\left[\begin{array}{ccc}
1 & 0 & 0 \\
0 & 1 & 0 \\
0 & 0 & 1 \\
-a & a & 0 \\
c-z & -1 & 0 \\
y & 0 & -b \\
a(a+c-z) & -a(a+1) & 0 \\
-(a+1)(c-z) & a(c-z)+1 & 0 \\
-(a+b) y & a y & b^{2}
\end{array}\right] .
$$

Since the matrix OM has a full-column rank, the system is observable. Hence, the weather system is both controllable and observable; therefore, the system is a minimum realization. 